\title{
Measuring the Imaginary Part of the Complex Magnetic Permeability of Thin Films Using Resonant and Non-resonant Automated Measuring Systems
}

\author{
Anton V. Burmitskikh \\ Institute of Engineering Physics and \\ Radio Electronics \\ Siberian Federal University \\ Laboratory of Electrodynamics \\ and Microwave Electronics \\ Kirensky Institute of Physics \\ Krasnoyarsk, Russian Federation \\ aburmitskikh@icloud.com \\ Sofya A. Kleshnina \\ Institute of Engineering Physics and \\ Radio Electronics \\ Siberian Federal University \\ Laboratory of Electrodynamics \\ and Microwave Electronics \\ Kirensky Institute of Physics \\ Krasnoyarsk, Russian Federation \\ sofya.antipckina@yandex.ru
}

\author{
Boris A. Belyaev \\ Institute of Engineering Physics and \\ Radio Electronics \\ Siberian Federal University \\ Laboratory of Electrodynamics \\ and Microwave Electronics \\ Kirensky Institute of Physics \\ Krasnoyarsk, Russian Federation \\ belyaev@iph.krasn.ru
}

\author{
Nikita M. Boev \\ Institute of Engineering Physics and \\ Radio Electronics \\ Siberian Federal University \\ Laboratory of Electrodynamics \\ and Microwave Electronics \\ Kirensky Institute of Physics \\ Krasnoyarsk, Russian Federation \\ nik88@inbox.ru
}

\begin{abstract}
This paper considers resonant and nonresonant methods for measuring magnetic characteristics of $\mathrm{Ni}_{80} \mathrm{Fe}_{20}$ thin films with $1000 \AA$ thickness. The imaginary part of complex magnetic permeability was measured by using resonant and nonresonant measuring cells. The resonant measuring cell included: a micro-strip line, a lumped capacitance, and an amplitude detector. The non-resonant measuring cell included a short-circuited micro-strip line. The studied sample of thin magnetic film is placed under the shortcircuited micro-strip line. The self-resonant frequency of the non-resonant measuring cell is higher than frequency of the ferromagnetic resonance (in field, equal 14 Oersteds). Ferromagnetic resonance method was used to study the change of measuring cell resonant frequency. The angular dependences of resonant frequency were achieved by the change of angle between constant magnetic field and high-frequency field of excitation, the hard axis of magnetization in thin magnetic film. The measurements were carried out using automation units. A numerical comparison of the obtained results showed that the difference between measurements does not exceed $5 \%$.
\end{abstract}

Keywords - thin magnetic films, magnetic permeability, ferromagnetic resonance.

\section{INTRODUCTION}

Thin magnetic films (TMF) made from soft magnetic materials have been widely used as active medium for the construction of various electrically-operated microwave devices, including tunable filters, delay lines, phase shifters, amplitude and phase modulators and nonlinear devices [1-2].
TMF are sensitive elements of magnetometers with weak quasi-stationary and high-frequency magnetic fields [3,4]. Comparison of the results obtained when measuring the imaginary part of the complex magnetic by the methods presented in this paper will allow determining of the magnetic characteristics of thin magnetic films more accurately. This will lead to the improved TMF based technical characteristics of devices.

\section{MEASUREMENT TECHNIQUES}

One of the most informative methods to study physical properties of magnetic materials is ferromagnetic resonance method (FMR) [5]. FMR is used to examine different ferromagnetics, including thin magnetic films. The FMR method is highly sensitive. It provides measurements of the magnetic characteristics in TMF with high precision. In practice, they usually conduct integral measurements of TMF samples by the FMR method, using the resonance and nonresonance measuring systems [6,7]. The directions of the magnetic fields in measuring systems are shown in Fig. 1.

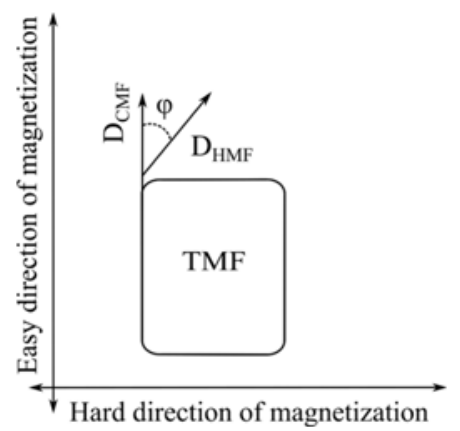

Fig. 1. The direction of the magnetic fields in the measuring system $\left(\mathrm{D}_{\mathrm{CMF}}\right.$ - direction of the constant magnetic field; $\mathrm{D}_{\mathrm{HMF}}$ - direction of the high-frequency excitation magnetic field) 
The resonance measuring cell (Fig. 2) includes: a microstrip line (MSL), a lumped capacitance, and an amplitude detector.

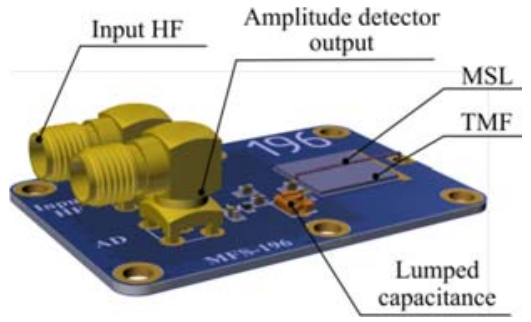

Fig. 2. The resonant measuring cell design

The resonance method uses a short-circuited microstrip line [8-9]. The sample of TMF is placed under the MSL. The MSL is the inductive part of resonant measuring cell (Fig. 2). The measurements in the resonant cell are carried out with the fixed amplitude constant magnetic field and the change of angle $\varphi$.

In the non-resonant method, the studied TMF sample is placed under the short-circuited microstrip line [10-12]. The self-resonant frequency of the non-resonant measuring cell (Fig. 3) is higher than FMR frequency (in fields up to hundreds of Oersteds) [13-15]. The measuring system is the load of the vector network analyzer, which operates in the required frequency range.

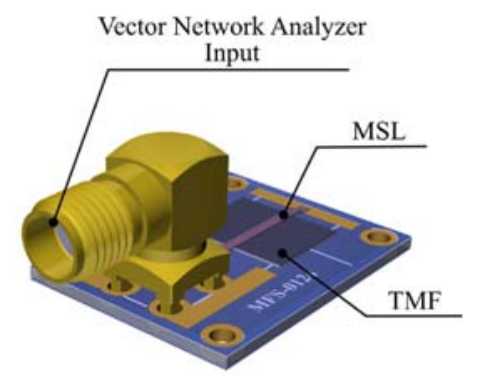

Fig. 3. The non-resonant measuring cell, designed to measure the magnetic characteristics of TMF

\section{MEASUREMENTS}

A sample of permalloy thin magnetic film was chosen as the object of research. The sample characteristics are as follows: composition $-\mathrm{Ni}_{80} \mathrm{Fe}_{20}$; number of layers -2 ; the thickness of each layer - $1000 \AA$.

Measuring cells with TMF sample were placed in the center of the Helmholtz coils. TMF in the measuring cells is placed between the metal strip and the metal screen. The magnitude of the constant magnetic field is $15 \mathrm{Oe}$. The resonant frequency of the measuring cell changed depending on the angle $\varphi$. The value of the resonant frequency was determined by Thomson equation. Fig. 4 shows the structural diagram of the resonant (b) and non-resonant (a) measuring systems.
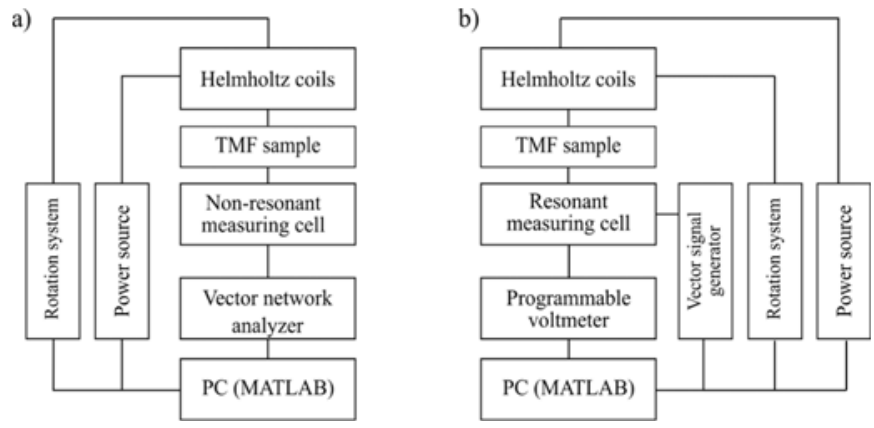

Fig. 4. The structural diagram of the non-resonant (a) and resonant (b) measuring units

When the TMF sample is introduced into the resonant measuring cell with a fixed value of capacitance and a change in the angle of rotation $\varphi$, the inductance of the measuring system changes. This reduces the resonant frequency of the measuring cell. To determine the resonant frequency of the measuring system, an automated unit was used (Fig. 5).

The unit consists of a vector signal generator (R\&S SMBV100A), a programmable voltmeter, a power source, Helmholtz coils, a rotation system, a resonant measuring cell with an amplitude detector, and a personal computer (Fig. 5).

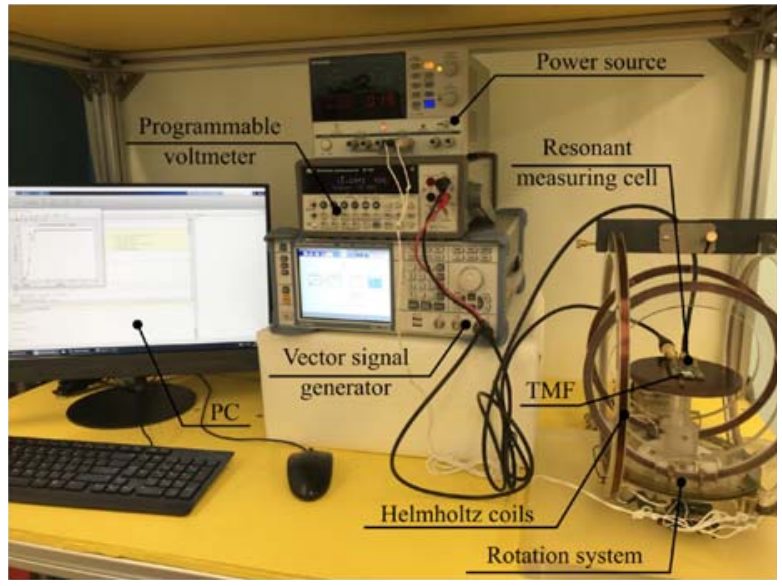

Fig. 5. The measuring unit for determining the imaginary part of the complex magnetic permeability, using a resonant measuring cell

Measurements in the non-resonant measuring cell (Fig. 3) were carried out using a vector network analyzer [16]. Frequency sweep of the microwave field change in the range from 50 to $2000 \mathrm{MHz}$. The angle $\varphi$ varied from $0^{\circ}$ to $90^{\circ}$ with $1{ }^{\circ}$ step. The directions of the fields in the measuring system corresponded to the circuit shown in Fig. 1. Automated measuring unit that allows measurements to be made by the non-resonant method using a vector network analyzer (R\&S ZVL) is shown in Fig. 6. 


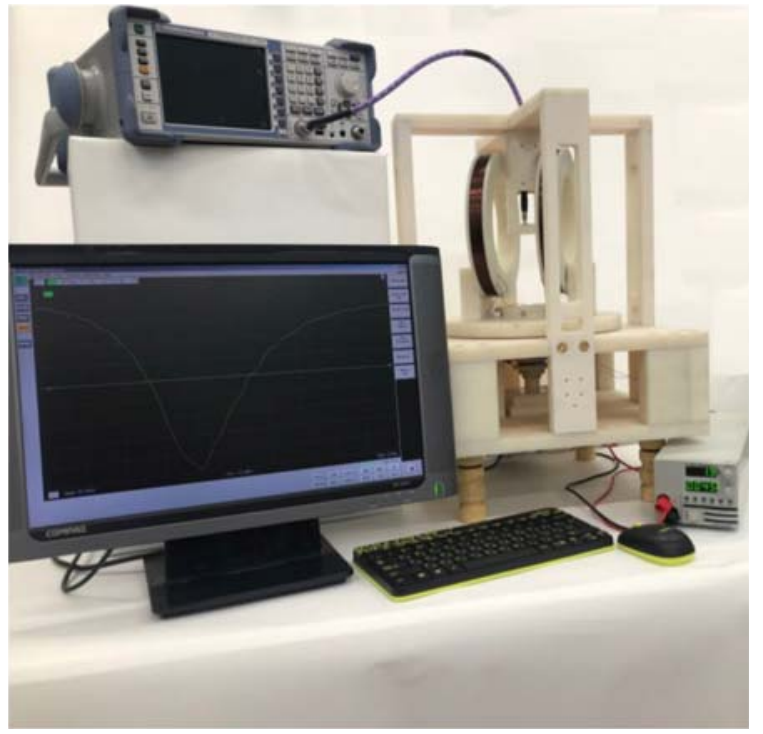

Fig. 6. The measuring unit for determining the imaginary part of the complex magnetic permeability, using a non-resonant measuring cell

\section{RESULTS}

The Q-factor of the resonance measuring cell at the frequency of $540 \mathrm{MHz}$ was measured when angle $\varphi$ was changed from $0^{\circ}$ to $90^{\circ}$ (Fig. 7).

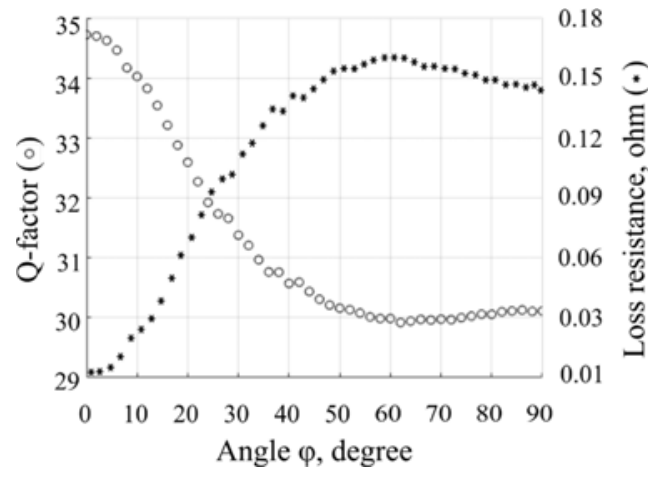

Fig. 7. Dependences of the quality factor and loss resistance in the resonant measuring cell on the angle $\varphi$

The measured inductance value of the resonant measuring cell without a TMF sample was $\sim 10 \mathrm{nH}$, and the capacitance value was $\sim 8 \mathrm{pF}$. The distribution of the value active resistance losses in the resonant measuring cell is shown in Fig. 7 (considering the value of losses in a system without TMF). The automated unit of the resonant measurement system searches for the resonant frequency by tuning the frequency of the microwave excitation generator and measuring the voltage value at the amplitude detector output. Using the method of the golden section and parabolic interpolation, the algorithm of the automated complex determines the maximum amplitude at the amplitude detector output. The system repeats operation algorithm for each angle $\varphi$. The resonant frequency value of the measuring cell from the angle of rotation $\varphi$ obtained by the automated system is shown in Fig. 8.

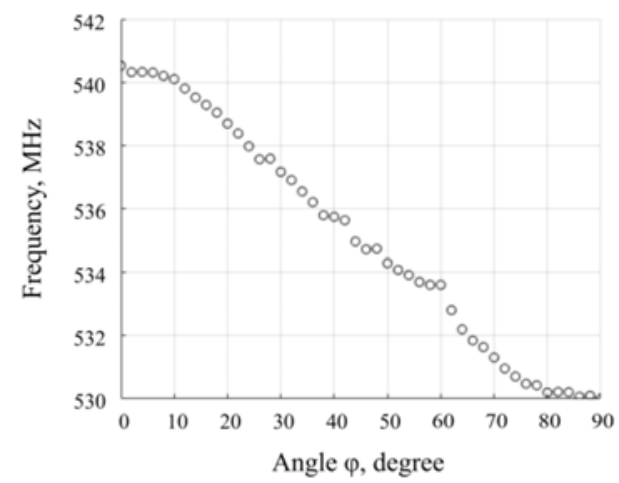

Fig. 8. The dependence of the frequency change of the resonant measuring cell on the angle $\varphi$

Before taking measurements using the non-resonant method the vector network analyzer was calibrated. During the calibration the measuring cell with the study sample (the sample was held by MSL without the use of fixing materials) was placed into a strong magnetic field. Strong magnetic field orientates domains in one direction. At this time the vector network analyzer is being calibrated. This allowed reducing the measurement error.

\section{CONCLUSION}

The imaginary part of the complex magnetic permeability was calculated considering the measured value of the complex reflection coefficient S11 of the measurement system. Fig. 9a shows the distribution of the imaginary part of the complex magnetic permeability $\mu$ ", measured using a non-resonant measuring cell. Fig. 9b shows the dependence of $\mu^{\prime \prime}$ on the angle $\varphi$ at the frequency of $530 \mathrm{MHz}$.

a)
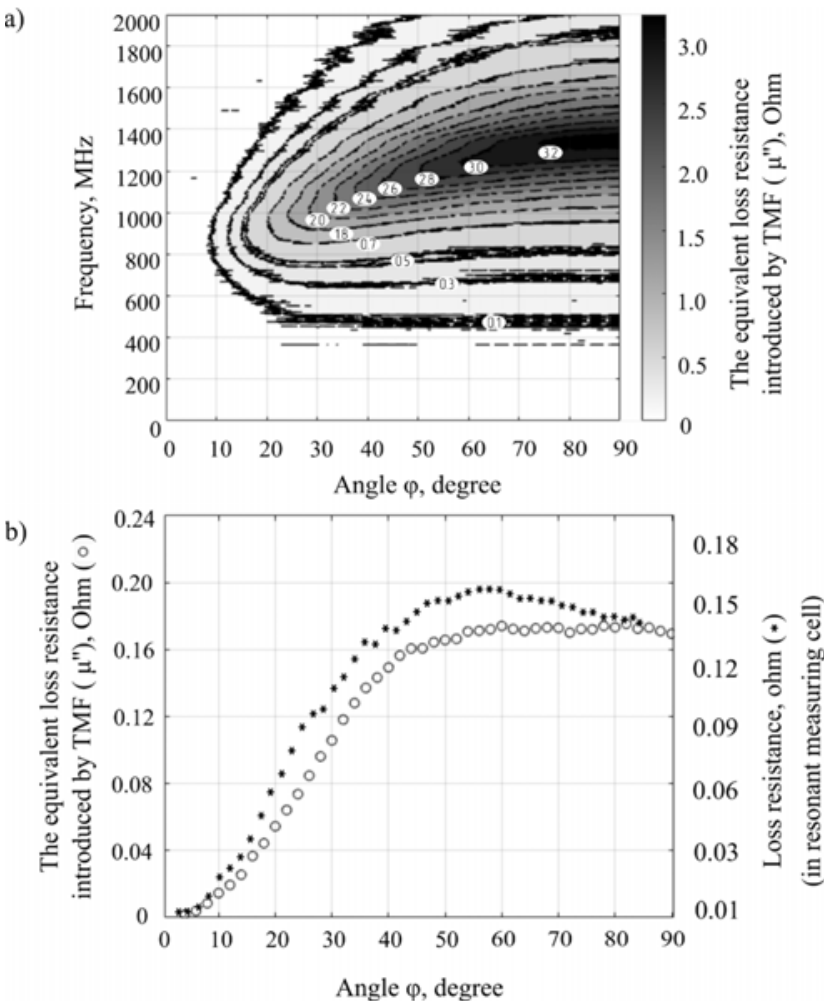

Fig. 9. The dependence of (a) the imaginary part of the complex magnetic permeability on the frequency and angle $\varphi$ in the TMF sample (nonresonant measuring method) (b) for the fixed value of excitation frequency $530 \mathrm{MHz}$, obtained by the non-resonant and the resonant methods 
According to the results of experimental data presented in Fig. $9 \mathrm{~b}$ it can be concluded that the imprecision between the data obtained using the resonant and non-resonant measuring cells does not exceed $5 \%$. In this case measurement time of one sample using resonant measuring cell at the fixed frequency and constant amplitude of magnetic field, sweep angle $\varphi$ - from $0^{\circ}$ to $90^{\circ}$, with $1^{\circ}$ step, is not more than 20 minutes. Under the conditions given for the resonance measurement method, the measurement time for one sample by the non-resonant method did not exceed 5 minutes.

Thus, the resonance measurement method exhibited its high sensitivity, no need for calibration and matching of the measuring cell, narrow bandwidth of the measurements. The non-resonance method demonstrated a wide range of measurements, the simplicity of the design of the measuring system, the ability to automate the measurement process and low sensitivity of the measuring system. The main goal of this work was to compare the results, obtained using resonant and non-resonant measuring cells.

\section{REFERENCES}

[1] B. A. Kalinikos, "Linear spin-waves devices," in Spin-Waves Devices, A. B. Ustinov, Ed. Moscow, Russia: Radiotechnics, 2013, pp. 89-100.

[2] Lee K.-S., "Conceptual design of spin wave logic gates based on a Mach-Zehnder-type spin wave interferometer for universal logic functions". Applied Physics Letters, vol. 104, 2008, doi: 10.1063/1.2975235.

[3] A. N. Babitskii, B. A. Belyaev, N. M. Boev, A. V. Izotov, "Low noise wideband thin-film magnetometer", in IEEE Sensors, 2017, pp. 316318.

[4] A. N. Babitskii, B. A. Belyaev, N. M. Boev, G. V. Skomorokhov, A. V. Izotov, R. G. Galeev, "Magnetometer of weak quasi-stationary and high-frequency fields on resonant microstrip transducers with thin magnetic films", Instruments and Experimental Techniques, vol. 59, pp. 425-432, 2016, doi: 10.1134/S0020441216030131.

[5] Ronald F. Soohoo, "Ferromagnetic resonance," in Magnetic Thin Films, R. V. Telesnina, World, Moscow, SSSR: Harper and Row, 1965, pp. 255-278.

[6] I. S. Maksymov, M. Kostylev, "Broadband stripline ferromagnetic resonance spectroscopy of ferromagnetic films, multilayers and nanostructures", Physica E Low-dimensional Systems and Nanostructures, vol. A247, pp. 254-293, 2015, doi: 10.1016/j.physe.2014.12.027.

[7] A. V. Burmitskikh, N. M. Boev, A. V. Izotov, V. G. Andyuseva, "Automated Measuring Unit for Analysis of Thin Magnetic Film Ferroresonance Spectrum", in 2019 International Siberian
Conference on Control and Communications (SIBCON), 2019, doi: 10.1109/SIBCON.2019.8729594.

[8] Y. Liu, L. Chen, C. Y. Tan, H. J. Liu, C. K. Ong, "Broadband complex permeability characterization of magnetic thin films using shorted microstrip transmission-line perturbation", Review of Scientific Instruments, vol. 76, no. 6, 2005, doi: 10.1063/1.1935429.

[9] S. N. Starostenko, K. N. Rozanov, A. V. Osipov, "A broadband method to measure magnetic spectra of thin films", Journal of Applied Physics, vol. 103, no. 7, 2008, doi: 10.1063/1.2832861.

[10] Yan Liu, L. Chen, C. Y. Tan, H. J. Liu, C. K. Ong, "Broadband complex permeability characterization of magnetic thin films using shorted microstrip transmission-line perturbation", Review of Scientific Instruments, vol. 76, 2005, doi: 10.1063/1.1935429.

[11] S. S. Kalarickal, P. Kvosik, Mingzhong Wu, C. E. Patton, M. L. Schneider, P. Kabos, T. J. Silva, J. P. Nibarger "Ferromagnetic resonance linewidth in metallic thin films: Comparison of measurement methods" Journal of Applied Physics, vol. 99, 2006, doi: 10.1063/1.2197087.

[12] C. Bilzer, T. Devolder, P. Crozat, C. Chappert, S. Cardoso and P. P. Freitas, "Vector network analyzer ferromagnetic resonance of thin films on coplanar waveguides: Comparison of different evaluation methods”, Journal of Applied Physics, vol. 101, № 7, 2007, doi: 10.1063/1.2716995.

[13] I. Neudecker, G. Woltersdorf, B. Heinrich, T. Okuno, G. Gubbiotti, C. H. Back, "Comparison of frequency, field, and time domain ferromagnetic resonance methods", Journal of Magnetism and Magnetic Materials, vol. 307, no. 1, pp. 148-156, 2006, doi: 10.1016/j.jmmm.2006.03.060.

[14] S. Azzawi, A. T. Hindmarch, D. Atkinson, "Magnetic damping phenomena in ferromagnetic thin-flms and multilayers", Journal of Physics D: Applied Physics, vol. 50, no. 47, 2017, doi: 10.1088/13616463/aa8dad.

[15] T. Lepetit, J. Neige, A-L. Adenot-Engelvin, M. Ledieu "Accurate Characterization of Both Thin and Thick Magnetic Films Using a Shorted Microstrip", IEEE Transactions on Magnetics (IEEE T $M A G N)$, vol. 50, no. 9, 2014, doi: 10.1109/TMAG.2014.2318022.

[16] J. Wei, H. Feng, Z. Zhu, Q. Liu, and J. Wang “A short-circuited coplanar waveguide to measure the permeability of magnetic thin films: Comparison with short-circuited microstrip line", Review of Scientific Instruments, vol. 86, no. 11, 2015, doi: 10.1063/1.4935499. 